\title{
Senior Solutions: Direct Composite Fixed Bridges
}

\author{
Ron Carlson* \\ Carlson Bridge Technologies, Inc., Hawaiia, USA
}

Received: September 07, 2017; Published: September 19, 2017

*Corresponding author: Carlson Bridge Technologies, Inc. Private Enterprise, Hawaiia, USA, Tel: 01-808-735-0282; E-mail: ddscarlson@hawaiiantel.net

\section{Introduction}

Remaining teeth in Americans according to the NIH's 2004 report state that only 22 out of 32 teeth per person are present in people 50 to 64 years of age. That computes to 10 missing teeth per person at 50 or above as the mean stat. It is also calculated that one tooth is missing in $69 \%$ of people 35 to 44 years of age. The true statistics of the condition of people's missing teeth will never really be known as our senior population continues to grow.

A less well know patient population that is underserved are those with special needs defined by federal, state and county agencies. Approximately 1 in 5 or 56.7 million Americans out of our current population have some form of disability. Many of these people do not receive dental care that they are in need of and require to maintain or elevate the quality of life. It is admitted by dental economists that there has been a loss in private practice base population with a downturn in busyness for the general dental practitioners. The dental schools are addressing this fact in the modification of their dental school programs looking at these social issues.

Middle class America has been greatly diminished in size and Starbuck knows this. Starbucks have just recently altered their business model based on the fact that the average purchaser has declined in their visits to the bar and their purchases of high-end items. If Starbucks is changing its business model, might we be wise to look at our own? We can glean from these observations that as we advance in years, with or without special needs, we also advance in tooth loss. If one has practiced decades one meets the obvious: people are continuously challenged with tooth loss and given our economic climate are hard pressed to seek treatment and pay for that care.

With this view in mind we assert that there are five ways to solve a missing tooth space issue and potential collapse of the dental arch integrity that may assist one in developing a new business model. We identify this model as follows with a new reality. This model is called the "Synergetic Model" which is founded upon:

1) effectiveness and efficiency

2) creativity in art and
3) Doing more with less.

As you will learn our system will give the dental practitioner "the mostest for the leastest" in respects, physiologically, structurally, artistically, remuneratively! First, one may choose to do nothing, which often leads to other more involved issues such as gum defects, rotation, hyper eruption and misalignment of existing teeth, leading to more tooth loss, nutritional challenges due to poor mastication, jaw joint issues, or muscular pain.

Or, one could construct a removable bridge the "flipper" method. This has its limitation in that it may loosen; losing its capacity to do the work of mastication thus damaging support tissues; collapse or breaking of the replacement, or it may be misplaced or lost.

Or, one could opt for the dental implant and it's attending challenges. Only $9 \%$ of the dentists in the America use this method. Cost of most single implants may about $\$ 4-8,000.00$. The downside, other than costs given what we know about our economy is bone rejection, peri-implantitis, or breakage of the implant, or systemic oral septicemia. Recently it was reported that there is a four times greater risk of implant rejection in people on anti-depressants the second most prescribed drug for people in America. It has also been reported by experts in the field of implant logy that the actual success rate for 3 to 5 years is about $61 \%$, not $96 \%$.

The fourth method available is the traditional fixed bridge for a missing tooth, requiring radical stripping away of outer enamel, the "enamel peel" cutting method, which may leaves one with a tooth stump, hastening its demise through gangrene. We present and offer the fifth method that, in most cases, can be done in one appointment of about an hour and a half, at a fraction of the cost of any other methods and requires little more than cleaning the support teeth surfaces and adhering the "Winged Pontic" into the space. Mostest for the leas test through synergy.

Many dental practitioners may be familiar with the composite fiber reinforced bridges advanced by some companies. Whether this methodology of fibers included in the matrix of composites works is equivocal and in our estimation and research the fibers interfere with the strength of the composite matrix. Three point 
bend tests show this to be so (See AGD 2003 Journal Knight and Whittaker). Ruling out the first option we show the advantages and disadvantages of the various methods of tooth replacement in table 1 . We trust that this view of the professions replacement work will give the younger dental practitioner an outline for his or her continuing success in private practice (Table 1).

Table 1: Single Tooth Replacement Comparison.

\begin{tabular}{|c|c|c|c|c|}
\hline Cost & $\sim \$ 1,740.00$ & $\sim \$ 4,500.00$ & $\sim \$ 6,400.00$ & $\sim \$ 1,600.00$ \\
\hline No. of Visits & $2-3$ & $3-5$ & $12-20$ & 1 \\
\hline Definitive Result & No & Yes & Yes & Yes \\
\hline Approx. Lifespan & 6mo.-1yr. & $3-5 y r s$ & $?$ & $3-5 y r s$ \\
\hline Tissue Altered & Little & Yes & Yes & None \\
\hline Completion time & 1-2 Weeks & About 1 month & 6 mo.-1 year & One Day \\
\hline Time in chair & 1 hour & 2-3 hours & 2-5 hours & 1.5 hour \\
\hline Expected pain & No & Moderate to High & Moderate to High & No \\
\hline Needed meds & No & Yes & Yes & No \\
\hline Easily modified & No & No & No & Yes \\
\hline Shade modified & No & No & No & Yes-Anytime \\
\hline Laboratory Costs & Yes & Yes-High & Yes-Very High & None \\
\hline Stress Doctor & Very little & Very High & Very High & Little/Moderate \\
\hline Stress Patient & Very little & Moderate/High & Very High & Little/None \\
\hline Death Threat & None & Moderate & Moderate to High & None \\
\hline
\end{tabular}

\section{Case Study}

An 82year old man split tooth \#12 and came to us to correct his missing tooth issue. He had heard of our tooth replacement process and wanted it done rather than extensive tooth reduction for a traditional bridge or implant placement for a crown. His physical condition prohibited long sessions in the chair since he had spinal scoliosis. In view of his requirements we accomplished the following steps as demonstrated in the photos as follow in one hour. In (Figure 1) a missing left first premolar, tooth \#12 is seen. The prefabricated "Winged Pontic" is seen in (Figure 2) having been adjusted for the edentulous space. Figure 3 shows occlusal relationships of the prefab "Winged Pontic," trimmed and adjusted into the space between the supports teeth without attachment composite affixed. The line of placement is confirmed as the "Winged Pontic" is adjusted to flow in and out of the space easily. Figure 4 shows the "Winged Pontic" first premolar \#12 with attachment composite applied to the proximal surfaces ready for insertion between \#11 and \#13, but only after preparation of the support teeth \#11 and \#13 for decay and surface cleanliness. After treating the enamel of support teeth \#11 and \#13 with etchant, water cleaning and drying, old existing composites are silanated with "Bond Enhancer and then a coat of clear resin and attachment composite are applied to proximal surfaces seen in (Figure 5). The prefab "Winged Pontic" (Figure 4) has first been etched for 30 seconds, cleaned with water, next treated with the $C B \circledR$ "WP" Bond Enhancer, clear resin is applied, followed by attachment composite application to proximal surfaces as seen in (Figure 4). The "Winged Pontic" is now ready for insertion between the support teeth seen in (Figure 5). The prefabricated, pretreated "Winged Pontic" is carried to the space and inserted as seen (Figure 5) and (Figure 6) in the predetermined line of draw. The attachment composite is smoothed over all aspects of the Pontic and support teeth and then light cured.

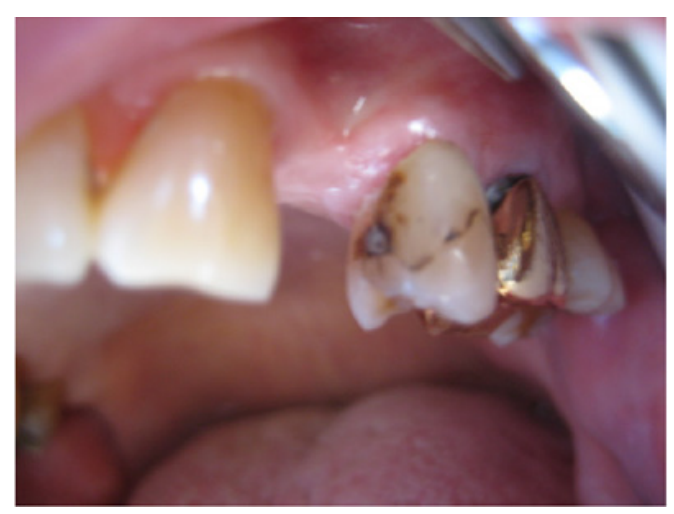

Figure 1: Pre-op showing slight decay on mesial of \#13 with a cervical composite restoration on the facial aspect.

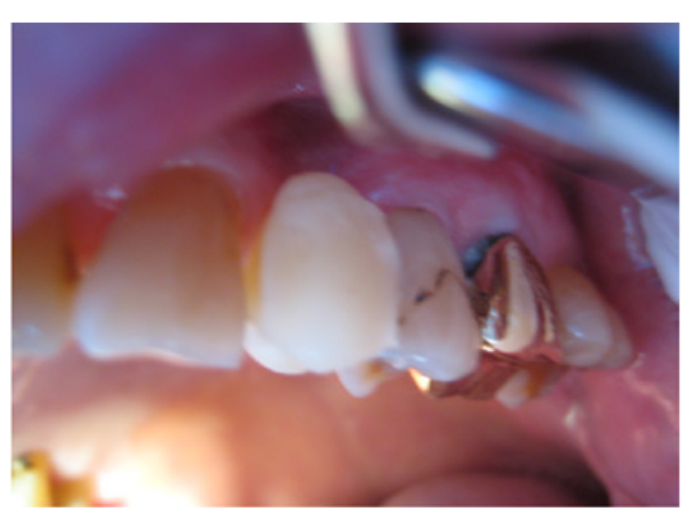

Figure 2: The "Winged Pontic" is shown after fitting into the edentulous area. 


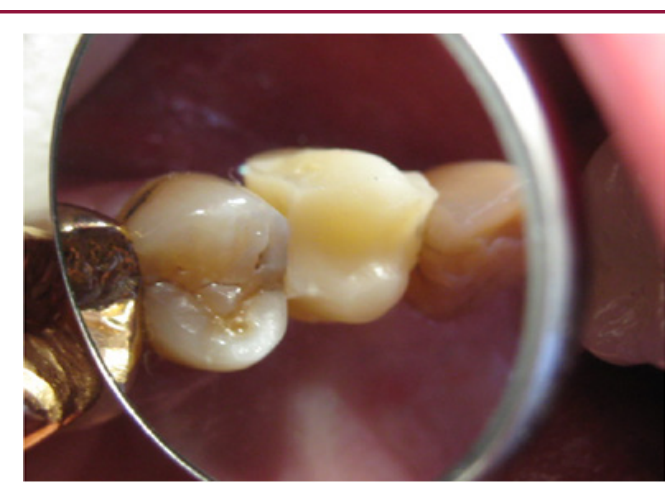

Figure 3: Occlusal view of loose fitting "Winged Pontic".

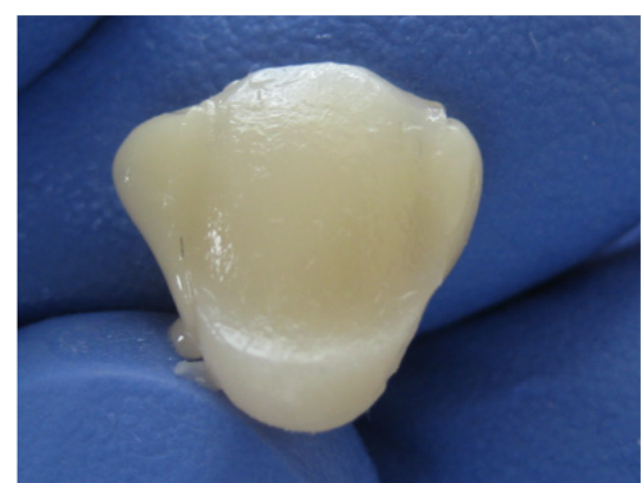

Figure 4: "Winged Pontic" prepared for installation with soft composite on proximals.

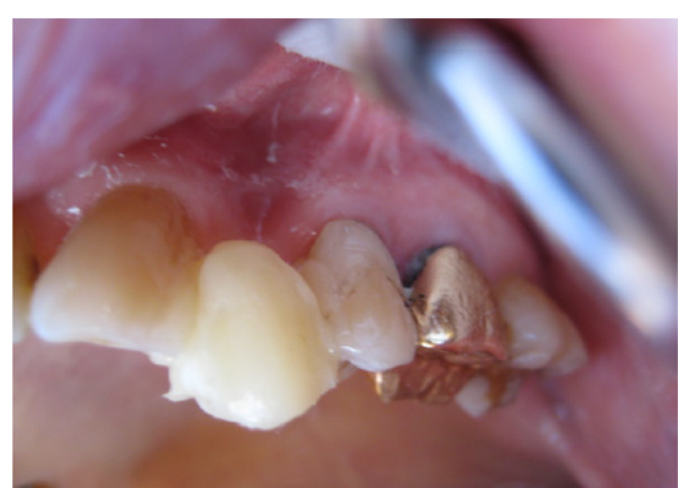

Figure 5: Once proximals of \#11 and \#13 are readied, the "WP" is installed.

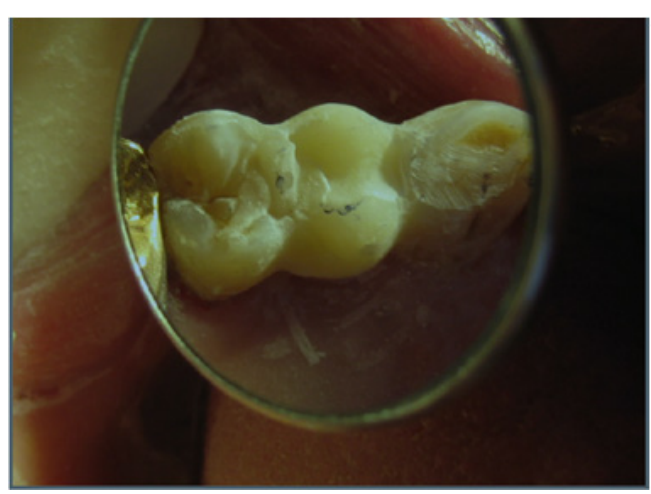

Figure 6: Once full insertion complete curing takes place and adjusting begins.
The "Winged Pontic" is layered over with finishing composite, if needed. This is a time when creative artistic skills may be applied. Once cured, the occlusion is checked and adjusted in all excursions. Various flame shaped finishing diamonds are used for characterization. Final polish may be done with fine finishing diamonds 25 micron and 50 micron, and rubber wheels. Final results are demonstrated in (Figures 7 \& 8).

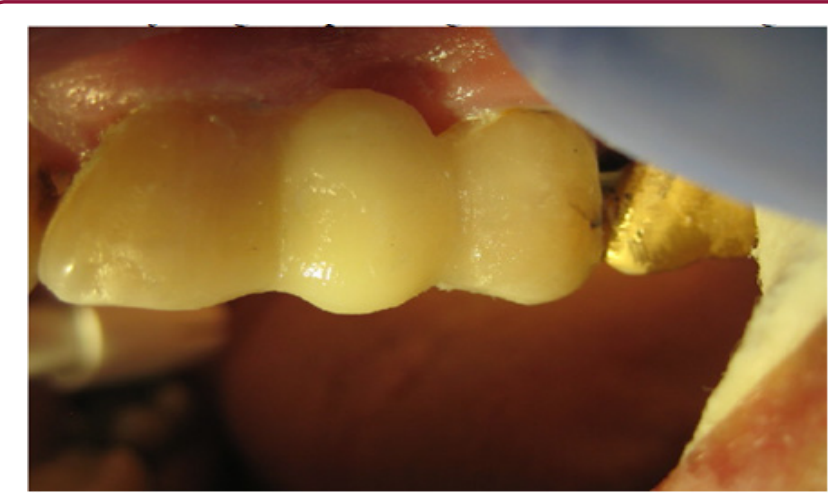

Figure 7: Final adjusting and polishing of the Carlson Bridge ${ }^{\circledR}$ "Winged Pointic."

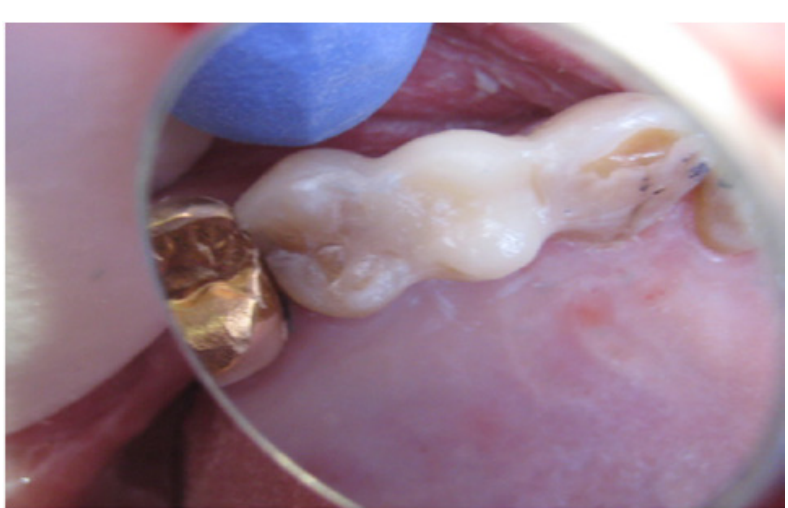

Figure 8: Occlusal view of the finsihed composite bridge.

\section{Summation}

This process, the Carlson Bridge ${ }^{\circledR}$ "Winged Pontic" tooth replacement system may open doors to new possibilities in the way dentists practice. Biosynthetic tissue engineering seems to be the leading edge in dentistry today, but only as an adjunct to other technical procedures carried out in the dental office, such as implants, flippers, or traditional porcelain veneer bridges. Resin composite materials are the closest to dentine and enamel in their strengths witnessed by their flexural modulus, compressive strengths and wear capacities With this new methodology, it may also be a pleasant experience for the patient who can shorten his or her time in the dental chair and come away with an immediate dental cosmetic enhancement. Additionally, it will not create a financial burden or, require extensive healing time or unnecessary oral discomfort. It is also beneficial to the dental practitioner since the procedure is shorter in duration, therefore physically less demanding, less complicated in that intricate tooth preparations are unnecessary, and ultimately more rewarding creatively, artistically and remuneratively. 
As with the new restorative composite systems of direct composite restorations the "Winged Pontic" prefab system offers a choice for the doctor and the patient regarding complex treatment plans and procedures. In this day and age of limited dental health budgets, it is an idea whose time has come. In senior populations health issues as well as costs are a major consideration in replacement of missing teeth. We have perfected the methodology for a one appointment, minimal time in the chair, direct composite bridge we identify as the Carlson Bridge ${ }^{\circledR}$ "Winged Pointic" tooth replacment system for one or more missing teeth. We present this short article with the intention of encouraging others to use this non-invasive, artistically satisfying and relatively inexpensive system not only for seniors, special needs people, but in young and middle age people as well (Figures 1-8).
Dr. RS Carlson graduated from the University Of Michigan School Of Dentistry in 1969 and completed Post Graduate training in pediatric dentistry with Strong-Carter Dental Clinic, Honolulu, Hawaii, 1970-1971. He is a founder of Kokua Kalihi Valley Dental Clinic in 1973 (http://www.kkv.net/index.php/history) and volunteered from 1973 to 1980 serving low-income families and immigrant populations from the South Pacific Islands and Asia. He has maintained a private practice in Honolulu since 1971 emphasizing Bio-Logical Dentistry. He can be reached at (808) 7350282, ddscarlson@hawaiiantel.net or carlsonbiologicaldentistry. com. Disclosure: Dr. Carlson is the inventor of the Carlson Bridge ${ }^{\circledR}$ "Winged Pontic" tooth replacement system, a noninvasive approach to replacing missing teeth, with patents issued in November 1999 and October 2001.

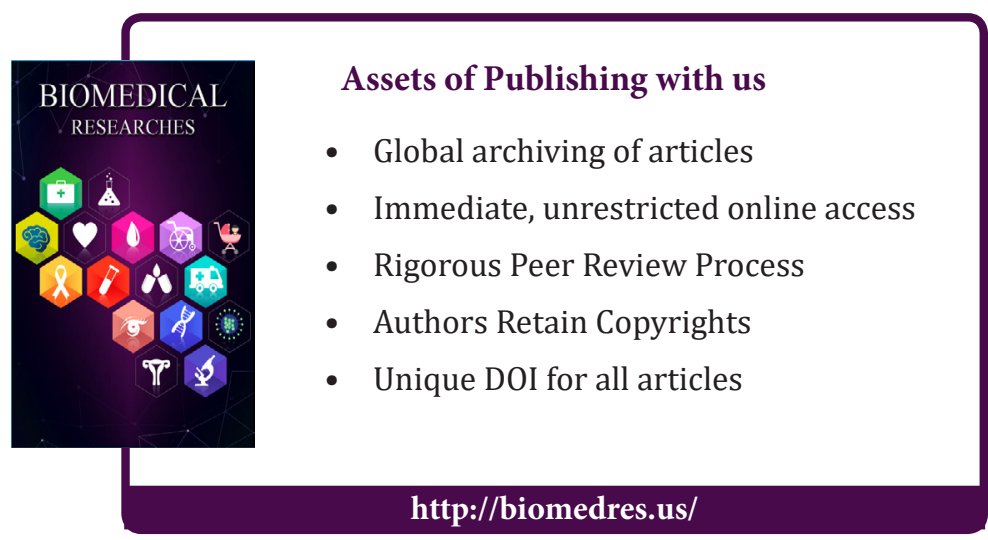

